\title{
The first decade of XFELs
}

X-ray science began 125 years ago with the image Wilhelm Röntgen took of his wife's hand. X-ray imaging quickly revolutionized medicine and science. Over the past century, increasingly sophisticated light sources have been developed, enabling scientists to 'see' the atomic and molecular structure of matter.

In the late 1950s, the by-product radiation from synchrotrons was first used in X-ray spectroscopy experiments. Free-electron lasers (FELs) were developed in the 1970s, but the first proposal to build an X-ray FEL (XFEL) came in 1992. From there, it took well over a decade until the first hard XFEL started operation in 2009, marking the beginning of a new era of $\mathrm{X}$-ray science.

The past decade has seen the commissioning, construction and opening of other FEL facilities ranging from vacuum ultraviolet (VUV) to soft and hard X-rays in Europe and Asia (see timeline; LCLS Linac Coherent Light Source, PAL Pohang Accelerator Laboratory, DCLS DaLian Coherent Light Source, SXFEL Shanghai XFEL). Today, a new generation of XFEL facilities, with higher repetition rates, is emerging.

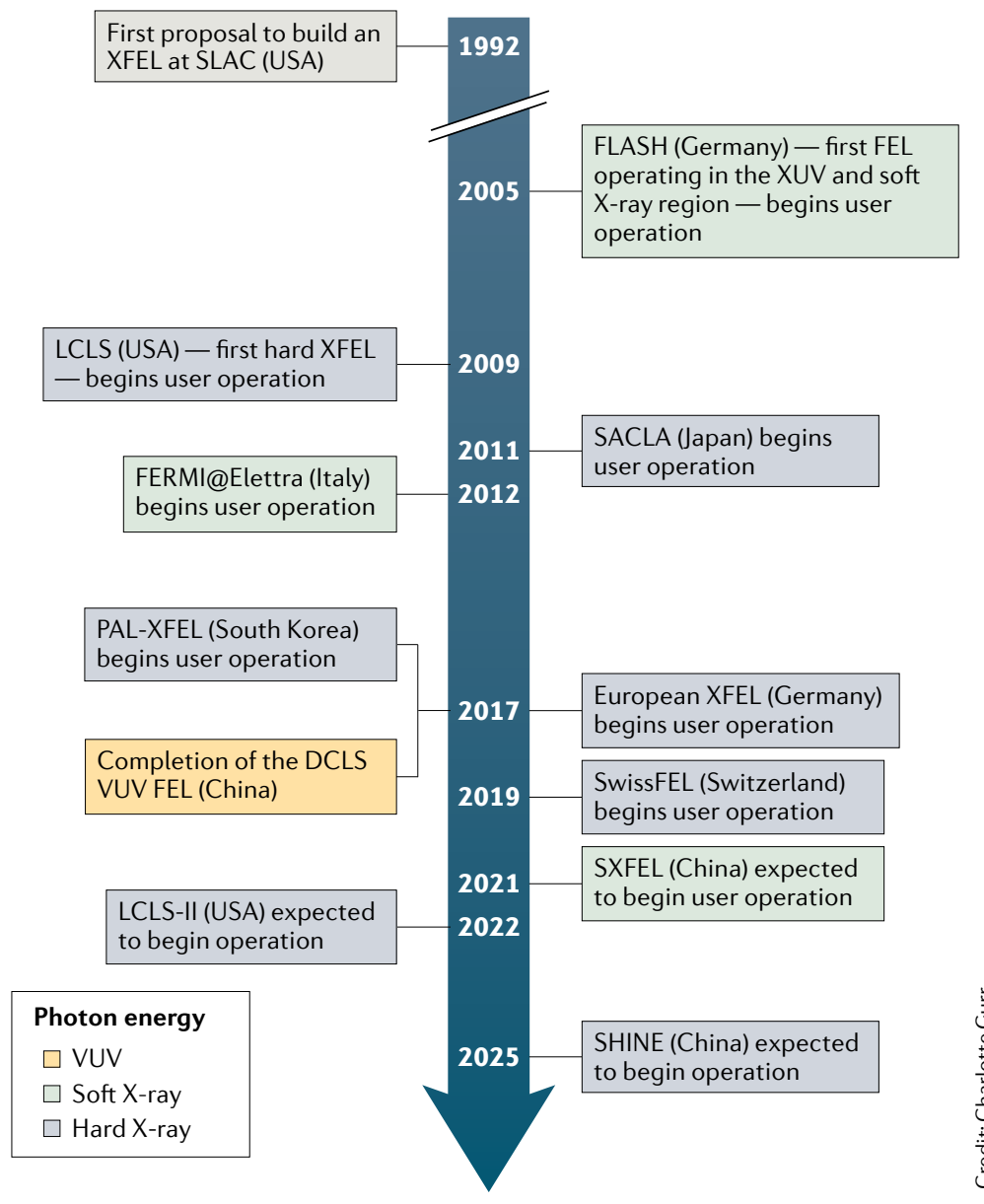

New facilities are expected to begin operation in the next 5 years in the USA and China, and the UK is considering the scientific case for an XFEL.

Iulia Georgescu 